\title{
RESÍDUOS VEGETAIS NA SUPERFÍCIE DO SOLO AFETAM A ACIDEZ DO SOLO E A EFICIÊNCIA DO HERBICIDA FLUMETSULAM
}

\author{
SOIL SURFACE RESIDUES AFFECT SOIL ACIDITY AND \\ FLUMETSULAM HERBICID EFFICIENCY
}

\author{
Antonio Sergio do Amaral ${ }^{1}$ Vitor Spader $^{2}$ Ibanor Anghinoni $^{3}$ \\ Egon Jose Meurer ${ }^{3}$
}

\section{RESUMO}

\begin{abstract}
Com o objetivo de avaliar o potencial de resíduos de duas espécies vegetais em alterar a acidez do solo e a atividade do herbicida flumetsulam, foi conduzido um experimento em casa de vegetação no Departamento de Solos da Universidade Federal do Rio Grande do Sul, em novembro de 1998. Amostras da camada superficial $(0-20 \mathrm{~cm})$ de um cambissolo húmico distrófico foram colocadas em vasos, com capacidade de 1,0kg, onde se aplicou na superfície: 0 , 5 e $10 t$ ha $^{-1}$ de resíduos de aveia-preta e de ervilhaca comum, com e sem aplicação do herbicida. Utilizouse aveia-branca como cultura reagente. $O$ delineamento experimental foi o inteiramente casualizado com três repetições. $O$ resíduo da ervilhaca aumentou o $\mathrm{pH}$ do solo e ambos os resíduos diminuíram o alumínio trocável na camada de $0-3 \mathrm{~cm}$ de profundidade, sendo o efeito da ervilhaca mais pronunciado. $O$ decréscimo do alumínio trocável foi relacionado a dois mecanismos: complexação pela matéria orgânica e aumento do $\mathrm{pH}$. Os resíduos vegetais aplicados sobre a superfície do solo aumentaram a eficiência do herbicida flumetsulam, especialmente quando o herbicida foi aplicado sobre os resíduos da leguminosa, que teve maior efeito na elevação do $\mathrm{pH}$ do solo.
\end{abstract}

Palavras-chave: resíduos vegetais, acidez, herbicida.

\section{SUMMARY}

An experiment was conducted in the greenhouse of the Soils Department of the Federal University of Rio Grande do Sul, in November of 1998, to evaluate the potential of two crop residues to modify the acidity of the soil and the activity of the flumetsulam herbicide. The experimental units consisted of pots containing an Haplumbrept soil from the surface layer $(0-20 \mathrm{~cm})$ in with it was applyed 0, 5 and $10 t \mathrm{ha}^{-1}$ of black oat and of common vicia residues, with and without the herbicide application in the soil surface, and arranged in a complete randomized design, with three replications. White oat was used as test culture. Vicia residues increase soil $\mathrm{pH}$ and both residues decreased exchangeable $A l$ in the $0-3 \mathrm{~cm}$ layer, with the effect of vicia being greater. The decrease of the exchangeable aluminum was related to both mechanisms: organic complexation and soil $\mathrm{pH}$ increase. Soil surface crop residues increased the herbicide efficiency, specially when this was applied over vicia residues, wich had greater increased in the soil $\mathrm{pH}$.

Key words: crop residues, acidity, herbicide.

\section{INTRODUÇÃO}

Geralmente, os solos ácidos das regiões tropicais e subtropicais apresentam $\mathrm{pH}$ baixo, teores de alumínio que podem ser fitotóxicos e baixos teores de cálcio e magnésio trocáveis. Essas características, que são desfavoráveis ao desenvolvimento da maioria das culturas, podem ser corrigidas pela calagem.

Resíduos vegetais deixados na superfície do solo podem diminuir a acidez e reduzir o efeito do alumínio (HUE \& AMIEN, 1989; MIYAZAWA et al., 1993). Entretanto, HOYT \& TURNER, 1975; HUE et al., 1986; MIYAZAWA et al., 1993, afirmam que não se conhece, exatamente, quais os mecanismos envolvidos nas reações de resíduos vege-

\footnotetext{
${ }^{1}$ Engenheiro Agrônomo, MSc., Aluno do Curso de Doutorado em Ciência do Solo, Programa de Pós-Graduação em Agronomia, Universidade Federal do Rio Grande do Sul (PPGA-UFRGS), Av. Bento Gonçalves, 7712, C.P. 776, 90001-970, Porto Alegre, RS. E-mail: asamaral@ hotmail.com. Autor para correspondência.

${ }^{2}$ Engenheiro Agrônomo, Aluno do Curso de Mestrado em Fitotecnia, PPGA-UFRGS.

${ }^{3}$ Engenheiro Agrônomo, Professor do Departamento de Solos da UFRGS. Bolsista CNPq.
} 
tais no solo. $\mathrm{O}$ efeito no $\mathrm{pH}$ do solo tem sido atribuído à adsorção de $\mathrm{H}^{+}$e $\mathrm{Al}^{+++}$na superfície do material vegetal (HOYT \& TURNER, 1975), à troca de ânions entre o $\mathrm{OH}^{-}$terminal de óxidos de $\mathrm{Fe}$ e $\mathrm{Al}$ e os ânions orgânicos (HUE \& AMIEN, 1989) e ao aumento do potencial de oxidação biológica de ânions orgânicos (HELYAR, 1991). A complexação do alumínio por substâncias húmicas da matéria orgânica parece ser a reação mais importante sob ponto de vista da redução da fitotoxidez, uma vez que essa reação tende a ser mais duradoura do que o efeito no pH do solo (SALET, 1998). É importante salientar que, segundo ERNANI \& GIANELLO, 1983, a elevação do $\mathrm{pH}$ acontece imediatamente após a adição do resíduo e que, com o tempo, tende a voltar ao seu valor inicial.

No sistema plantio direto, a massa seca da parte aérea dos diferentes resíduos vegetais permanece na superfície do solo. Os herbicidas são intensamente utilizados nesse sistema e são aplicados diretamente sobre os resíduos vegetais na superfície do solo, mas a ação dos resíduos vegetais na eficiência dos herbicidas tem sido pouco avaliada. A alteração no estado de ionização do herbicida com a variação do pH do solo influi na sua adsorção aos colóides orgânicos e minerais do solo, o que afeta a degradação, persistência e, principalmente sua atividade, pois menor quantidade de herbicida poderá estar disponível para absorção pelas raízes das plantas (BAYLEY \& WHITE, 1970; KHAN, 1978; LOCKE \& BRYSON, 1997). O flumetsulam, conforme FONTAINE et $\boldsymbol{a l}$., 1991; MURPHY \& SHAW, 1997, apresenta caráter de ácido fraco e sua atividade aumenta com a elevação do $\mathrm{pH}$ do solo por diminuir a sua adsorção na superfície dos colóides.

Espera-se, que os resíduos vegetais deixados na superfície do solo no sistema plantio direto, elevem o valor do $\mathrm{pH}$ do solo e, por consequência, a eficiência do herbicida.

Assim, os objetivos deste trabalho foram avaliar o potencial de resíduos de duas espécies vegetais em alterar a reação do solo e avaliar a eficiência do herbicida flumetsulam aplicado na superfície do solo sobre diferentes doses desses resíduos.

\section{MATERIAL E MÉTODOS}

O experimento foi conduzido na casa de vegetação no Departamento de Solos da Universidade Federal do Rio Grande do Sul (UFRGS), em novembro de 1998. Utilizou-se resíduo vegetal das espécies: aveia-preta (Avena strigosa) e ervilhacacomum (Vicia sativa). Amostras da parte aérea dessas plantas foram coletadas logo após o pleno flores- cimento, na Estação Experimental Agronômica (EEA) da UFRGS, no município de Eldorado do Sul (RS); foram secas em estufa a $65^{\circ} \mathrm{C}$ por 48 horas, moídas e passadas em peneiras de $1,0 \mathrm{~mm}$. Os resíduos de ervilhaca apresentaram, em média, 0,51\% de $\mathrm{Ca}, 0,18 \%$ de $\mathrm{Mg}$ e $1,96 \%$ de $\mathrm{K}$, e os de aveia $0,27 \%, 0,15 \%$ e $1,14 \%$ respectivamente, para os mesmos elementos.

Utilizaram-se amostras da camada superficial $(0-20 \mathrm{~cm})$ de um cambissolo húmico distrófico, textura franco argilosa. O solo foi seco ao ar, peneirado em malha de $2 \mathrm{~mm}$ e adicionado $2,5 \mathrm{~g}$ de nitrogênio, $40 \mathrm{~g}$ de fósforo e $19,8 \mathrm{~g}$ de potássio por $\mathrm{kg}$ de solo, quantidades equivalente às recomendações (COMISSÃO..., 1995) para a cultura da aveiabranca (Avena sativa), utilizando-se uréia, superfosfato triplo e cloreto de potássio como fontes para os respectivos nutrientes. $\mathrm{O}$ solo apresentava, antes da adição dos fertilizantes: $\mathrm{pH}\left(\mathrm{H}_{2} \mathrm{O}\right) 3,9$; necessidade de calcário (método SMP) de 21t ha' $; 43 \mathrm{~g} \mathrm{~kg}^{-1}$ de matéria orgânica (combustão úmida); $4,4 \mathrm{mg} \ell^{-1} \mathrm{de}$ fósforo e $62 \mathrm{mg} \ell^{-1}$ de potássio dispóníveis (MehlichI); $6,0 \mathrm{cmol}_{\mathrm{c}} \mathrm{dm}^{-3}$ de alumínio e $2,2 \mathrm{cmol}_{\mathrm{c}} \mathrm{dm}^{-3} \mathrm{de}$ cálcio+magnésio trocáveis $(\mathrm{KCl} 1 \mathrm{M})$.

As unidades experimentais consistiram de vasos plásticos contendo um $\mathrm{kg}$ de solo seco, sobre o qual se adicionaram os seguintes tratamentos: doses equivalentes a 0 (zero), 5 (cinco) e 10 (dez) $\mathrm{t} \mathrm{ha}^{-1} \mathrm{de}$ resíduos de aveia e de resíduos de ervilhaca, com e sem aplicação do herbicida flumetsulam. A testemunha caracterizou-se pela dose zero de qualquer resíduo e sem aplicação do herbicida. Os resíduos foram aplicados na superfície do solo. Sete dias após a adição dos resíduos, semearam-se oito sementes de aveia-branca (Avena sativa) por vaso, sendo, posteriormente, reduzidas a quatro plantas por vaso. A seguir, aplicou-se o herbicida na dose equivalente a $28 \mathrm{~g} \mathrm{ha}^{-1}$, utilizando um pulverizador costal pressurizado com $\mathrm{CO}_{2}$. A umidade do solo foi mantida próximo à capacidade de campo. $\mathrm{O}$ delineamento experimental utilizado foi o inteiramente casualizado, com três repetições. Para a comparação de médias, utilizou-se o teste de Tukey com nível de significância de $5 \%$.

No solo, determinou-se o pH em água (1:1) em amostras coletadas na profundidade de 0-3 $\mathrm{cm}$, aos 7 e 21 dias após a adição dos resíduos e no final do experimento, aos 28 dias. O alumínio trocável, extraído com $\mathrm{KCl} 1 \mathrm{~mol} \mathrm{dm}{ }^{-3}$ ou com $\mathrm{CuCl}_{2}$ $0,5 \mathrm{~mol} \mathrm{dm}^{-3}$, foi determinado em amostras coletadas na mesma profundidade, somente no final do experimento. A contribuição de mecanismos de controle da disponibilidade de alumínio no solo foi calculada segundo MIYAZAWA et al. (1993) por: 
$\Delta / \mathrm{Al}=\mathrm{Al}\left(\mathrm{CuCl}_{2}\right)-\mathrm{Al}(\mathrm{KCl})$.

$\mathrm{Al}($ Complexo orgânico $)=\Delta / \mathrm{Al}$ (tratamento) $-\Delta / \mathrm{Al}$ (testemunha)

Contribuição complexo orgânico $=\frac{\mathrm{Al}(\text { complexo orgânico })}{\mathrm{Al} \mathrm{KCl}(\text { testemunha })-\mathrm{Al} \mathrm{KCl} \mathrm{(tratamento)}} \times 100$

Contribuição do $\mathrm{pH}=100$ - contribuição complexo orgânico:

onde $\Delta \mathrm{Al}$ é a diferença da quantidade de alumínio extraído pelo $\mathrm{CuCl}_{2}$ e pelo $\mathrm{KCl}$; $\mathrm{Al}$ (Complexo orgânico) é a quantidade de alumínio complexado pelas substâncias húmicas.

As análises químicas foram feitas segundo descrito por TEDESCO et al.(1995), exceto a extração do alumínio com $\mathrm{CuCl}_{2} 0,5 \mathrm{~mol} \mathrm{dm}^{-3}$, que foi realizada segundo descrito por SALET (1998). Aos 28 dias, encerrou-se o experimento, quando foram colhidas as quatro plantas de aveia-branca por vaso, separando-se a parte aérea das raízes, que após secas em estufa a $60^{\circ} \mathrm{C}$ até peso constante, determinou-se o peso da massa seca. $\mathrm{O}$ fator tempo não foi considerado na análise estatística dos dados obtidos.

\section{RESULTADOS E DISCUSSÃO}

$\mathrm{O}$ pH do solo foi afetado significativamente pela interação doses de resíduos versus tipo de resíduo. A aplicação do herbicida flumetsulam não afetou o $\mathrm{pH}$ do solo, independentemente do tipo de resíduo ou da dose utilizada.

Os resíduos de aveia preta modificaram significativamente o $\mathrm{pH}$ do solo em relação à testemunha (sem resíduos e sem herbicida) notadamente no tratamento onde foi aplicado $10 \mathrm{tha}^{-1}$. Os resíduos de ervilhaca, já na avaliação realizada no sétimo dia, elevaram o $\mathrm{pH}$ do solo na profundidade de $0-3 \mathrm{~cm}$ em qualquer dose utilizada (tabela 1). Isso indica

Tabela 1 - Valores de pH do solo (1:1) das amostras de um cambissolo húmico na profundidade de $0-3 \mathrm{~cm}$, após a adição de resíduos de aveia-preta e ervilhaca comum.

\begin{tabular}{|c|c|c|c|c|c|c|c|c|c|}
\hline \multirow{3}{*}{ Resíduos } & \multicolumn{3}{|c|}{7 dias } & \multicolumn{3}{|c|}{21 dias } & \multicolumn{3}{|c|}{28 dias } \\
\hline & \multicolumn{9}{|c|}{ Doses $\left(\mathrm{t} \mathrm{ha}^{-1}\right)$} \\
\hline & 0 & 5 & 10 & 0 & 5 & 10 & 0 & 5 & 10 \\
\hline
\end{tabular}

.pH do solo

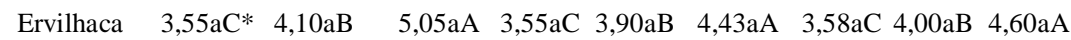

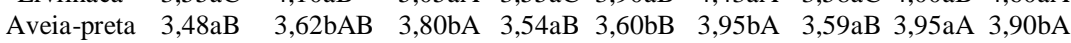

* Médias seguidas de mesma letra minúscula na coluna e maiúscula na linha, dentro de cada época de avaliação, não diferem pelo teste de Tukey em nível de 5\% de probabilidade de erro. uma reação rápida dos resíduos da ervilhaca nos primeiros dias de sua aplicação sobre o solo. Esse efeito é semelhante ao da aplicação superficial de calcário no sistema plantio direto consolidado e se manifesta rapidamente nas características químicas, tanto da solução como da fase sólida do solo (AMARAL, 1998). A elevação do pH do solo pode ser atribuída à adsorção do $\mathrm{H}^{+}$nos grupos funcionais de superfície dos compostos orgânicos dos resíduos vegetais (HOYT \& TURNER,1975; MIYAZAWA et $\boldsymbol{a l} .$, 1993) e ao potencial de oxidação biológica de ânions orgânicos, que são liberados em maior quantidade no início da decomposição dos resíduos (HELYAR, 1991). A intensidade dos efeitos na reação do solo tem sido relacionada às características e composição do material vegetal utilizado, sendo que os resíduos de leguminosas, geralmente, têm efeito mais pronunciado do que os de gramíneas (HUE \& AMIEN, 1989; MIYAZAWA et al., 1993). Esses efeitos podem estar relacionados aos maiores teores de cálcio, magnésio e potássio no tecido das leguminosas, que estariam associados a um maior teor de ânions orgânicos produzidos para a manutenção da neutralidade elétrica na planta (HELYAR, 1991).

Os resíduos vegetais de ambas as espécies associados ou não ao herbicida causaram decréscimos significativos nos teores de alumínio extraídos com $\mathrm{KCl}$ e com o $\mathrm{CuCl}_{2}$, verificando-se para estas variáveis interação tríplice significativa entre resíduos, doses de resíduos e herbicida (tabela 2). A solução de $\mathrm{CuCl}_{2} 0,5 \mathrm{~mol} \mathrm{dm}{ }^{-3}$ extraiu mais alumínio que a solução de $\mathrm{KCl}$ $1 \mathrm{~mol} \mathrm{dm}^{-3}$ porque, além do alumínio trocável, também extrai o alumínio ligado especificamente a compostos de matéria orgânica do solo. $\mathrm{O}$ íon $\mathrm{Cu}$ é eficiente para extrair o alumínio adsorvido especificamente por deslocar outros metais ligados aos compostos da matéria orgânica devido a sua maior afinidade e ao $\mathrm{pH}$ baixo da solução (JUO \& KAMPRATH, 1979; HARGROVE \& THOMAS, 1981; SALET, 
Tabela 2 - Teores de alumínio na profundidade 0-3 cm extraído com $\mathrm{KCl}$ e $\mathrm{CuCl}_{2}$, num cambissolo húmico submetido à aplicação de doses de resíduos de aveia-preta e ervilhaca comum sem e com aplicação de herbicida.

\begin{tabular}{|c|c|c|c|c|c|c|}
\hline \multirow[b]{2}{*}{ Herbicida } & \multicolumn{3}{|c|}{ Resíduos de aveia $\left(\mathrm{t} \mathrm{ha}^{-1}\right)$} & \multicolumn{3}{|c|}{ Resíduos de ervilhaca $\left(\mathrm{t} \mathrm{ha}^{-1}\right)$} \\
\hline & 0 & 5 & 10 & 0 & 5 & 10 \\
\hline
\end{tabular}

\begin{tabular}{|c|c|c|c|c|c|}
\hline \multirow{3}{*}{$\begin{array}{l}\text { Sem } \\
\text { Com }\end{array}$} & \multicolumn{5}{|c|}{. } \\
\hline & $64,37 \mathrm{aA} *$ & $53,51 \mathrm{aB}$ & $50,06 \mathrm{aB}$ & $60,93 \mathrm{aA}$ & $34,10 \mathrm{aB} \quad 22,67 \mathrm{aC}$ \\
\hline & $63,35 \mathrm{aA}$ & $53,97 \mathrm{aB}$ & $48,63 \mathrm{aB}$ & $60,90 \mathrm{aA}$ & $27,43 \mathrm{bB} \quad 28,13 \mathrm{bB}$ \\
\hline \multicolumn{6}{|c|}{ 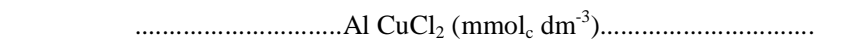 } \\
\hline Sem & $110,75 \mathrm{aA}$ & $108,93 \mathrm{aA}$ & $108,47 \mathrm{aA}$ & $111,39 \mathrm{aA}$ & $96,16 \mathrm{aB} \quad 93,43 \mathrm{aB}$ \\
\hline Com & $110,00 \mathrm{aA}$ & $111,21 \mathrm{aA}$ & $102,09 \mathrm{bB}$ & $110,75 \mathrm{aA}$ & $98,45 b B \quad 89,78 b C$ \\
\hline
\end{tabular}

*Médias seguidas de mesma letra minúscula na coluna e maiúscula na linha, dentro de cada tipo de resíduo, não diferem pelo teste de Tukey em nível de $5 \%$ de probabilidade de erro.

1998). Entre as espécies, a ervilhaca foi a que provocou maior decréscimo nos teores de alumínio, reduzindo o alumínio trocável em até $38,26 \mathrm{mmol}_{\mathrm{c}}$ $\mathrm{dm}^{-3}$, quando aplicado na dose de $10 \mathrm{t} \mathrm{ha}^{-1}$ sem herbicida. A aveia preta, para esta mesma dose, reduziu o alumínio em até $14,31 \mathrm{mmol}_{\mathrm{c}} \mathrm{dm}^{-3}$.

De acordo com os critérios propostos por MIYAZAWA et al. (1993), a contribuição dos mecanismos segundo os quais as duas espécies reduziram os teores do alumínio, também apresentaram resultados diferentes (tabela 3). Para a gramínea, o mecanismo de complexação do alumínio seria responsável por cerca de $80 \%$ da redução dos teores do elemento no solo e à variação do pH em $20 \%$. Para a leguminosa, a contribuição dos dois mecanismos, foi similar na dose menor, e mais acentuado para o mecanismo de complexação, na maior dose. Para a aveiapreta, a menor contribuição relativa do $\mathrm{pH}$ na diminuição do alumínio do solo é explicada pelo seu menor efeito na mudança do $\mathrm{pH}$ do solo. A ervilhaca, igualmente, foi mais eficiente do que a aveia-preta na complexação do alumínio, complexando 24,0 $\mathrm{mmol}_{\mathrm{c}} \mathrm{dm}^{-3}$ deste elemento, na maior dose, contra $11,7 \mathrm{mmol}_{\mathrm{c}}$ $\mathrm{dm}^{-3}$ para a aveia-preta.

A aplicação do herbicida flumetsulam, apesar de não ter afetado o $\mathrm{pH}$ do solo, afetou a planta reagente cultivada, pois o rendimento de matéria seca das raízes e da parte aérea da aveia branca foram significativamente menores com a aplicação do herbicida (tabelas 4 e 5). Verificou-se interação tríplice significativa entre resíduos, doses de resíduos e herbicida para a matéria seca de raízes (tabela 4) e apenas efeito isolado do herbicida para a variável matéria seca da parte aérea (tabela 5). Conforme FONTAINE et al. (1991), o maior efeito do flumetsulam ocorre no sistema radicular das plantas sensíveis, porém, neste trabalho, observou-se também efeito na parte aérea da planta testada.

A espécie vegetal e a dose dos resíduos vegetais afetaram a ação do herbicida. O herbicida foi mais eficiente quando aplicado sobre os resíduos da ervilhaca, reduzindo os rendimentos da parte aérea e das raízes da aveia-branca. Quando aplicado sobre os resíduos da aveia-preta, entretanto, o seu efeito foi muito menos pronunciado. Estes efeitos estão de acordo com a modificações no $\mathrm{pH}$ provocados pelos dois resíduos: o efeito da leguminosa foi mais acentuado. Herbicidas acídicos, como o Flumetsulam, com a elevação do pH do solo comportam-se como ânions devido a desprotonação de suas moléculas. Isto reduz a sua adsorção no solo, o que aumenta a sua disponibilidade resultando numa maior absorção pelas raízes das plantas, causando maior injúria nas espécies mais sensíveis (KLESCHICK et al., 1992; FONTAINE et al., 1991; MURPHY \& SHAW, 1997).

Tabela 3 - Quantidades de alumínio do cambissolo húmico submetido à aplicação de doses de aveia-preta e ervilhaca comum extraídas na profundidade $0-3 \mathrm{~cm}$, pelo $\mathrm{KCl}$ e pelo $\mathrm{CuCl}_{2}$, e valores de $\Delta$ - Al e Al-C.org. e contribuição relativa dos complexos orgânicos e do pH na redução do alumínio.

\begin{tabular}{|c|c|c|c|c|c|c|}
\hline \multirow[b]{2}{*}{ Tratamento } & \multicolumn{4}{|c|}{ Alumínio } & \multicolumn{2}{|c|}{ Contribuição } \\
\hline & $\mathrm{KCl} 1 \mathrm{~mol} \mathrm{dm}^{-3}$ & $\mathrm{CuCl}_{2} 0,5 \mathrm{~mol} \mathrm{dm}{ }^{-3}$ & $\Delta-\mathrm{Al}$ & Al-C.org. & Comp. org. & $\mathrm{pH}$ \\
\hline & \multicolumn{4}{|c|}{ 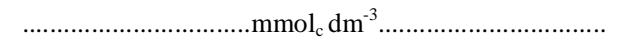 } & \multicolumn{2}{|c|}{$\ldots \ldots \ldots \ldots \ldots \ldots \ldots$} \\
\hline Testemunha & 64,4 & 111,0 & 46,6 & - & - & - \\
\hline $5 \mathrm{t}$ aveia-preta & 53,5 & 108,9 & 55,4 & 8,8 & 80,9 & 19,1 \\
\hline $10 \mathrm{t}$ aveia-preta & 50,1 & 108,5 & 58,4 & 11,7 & 82,3 & 17,7 \\
\hline 5 t ervilhaca & 34,1 & 96,2 & 62,1 & 15,4 & 51,0 & 49,0 \\
\hline 10 t ervilhaca & 22,7 & 93,4 & 70,7 & 24,1 & 57,8 & 42,2 \\
\hline
\end{tabular}

Ciência Rural, v. 30, n. 5, 2000. 
Tabela 4 - Rendimento de matéria seca de raízes $\left(\mathrm{g}\right.$ vaso $\left.^{-1}\right)$ da planta teste (aveia branca) num cambissolo húmico submetido à aplicação de doses de resíduos de aveiapreta e ervilhaca com e sem herbicida flumetsulam.

\begin{tabular}{|c|c|c|c|}
\hline \multirow[t]{2}{*}{ Resíduos } & \multicolumn{3}{|c|}{ Doses $\left(\mathrm{t} \mathrm{ha}^{-1}\right)$} \\
\hline & 0 & 5 & 10 \\
\hline & \multicolumn{3}{|c|}{..Com herbicida.. } \\
\hline Aveia-preta & $0,147 \mathrm{aA} *$ & $0,163 \mathrm{aA}$ & $0,113 \mathrm{aA}$ \\
\hline \multirow[t]{2}{*}{ Ervilhaca } & $0,148 \mathrm{aA}$ & $0,074 \mathrm{bB}$ & $0,097 \mathrm{bB}$ \\
\hline & \multicolumn{3}{|c|}{ Sem herbicida.. } \\
\hline Aveia-preta & $0,270 \mathrm{aA}$ & $0,260 \mathrm{aA}$ & $0,300 \mathrm{aA}$ \\
\hline Ervilhaca & $0,271 \mathrm{aB}$ & $0,280 \mathrm{aB}$ & $0,439 \mathrm{bA}$ \\
\hline
\end{tabular}

* Médias seguidas de mesma letra minúscula na coluna e maiúscula na linha não diferem pelo teste de Tukey em nível de 5\% de probabilidade de erro.

Tabela 5 - Rendimento de matéria seca da parte aérea da planta teste (aveia branca) em função do efeito isolado do herbicida (independentemente do tipo de resíduo e das doses).

\begin{tabular}{cc}
\hline Herbicida & Parte aérea $\left(\right.$ g vaso $\left.^{-1}\right)$ \\
\hline Sem & $0,409 \mathrm{a}^{*}$ \\
Com & $0,164 \mathrm{~b}$ \\
\hline
\end{tabular}

*Médias seguidas de mesma letra minúscula na coluna não diferem pelo teste de Tukey em nível de $5 \%$ de probabilidade de erro.

\section{CONCLUSÕES}

Os resíduos da ervilhaca aplicados sobre a superfície aumentaram o $\mathrm{pH}$ do solo (cambissolo húmico distrófico) na camada de $0-3 \mathrm{~cm}$ de profundidade; os resíduos da aveia-preta tiveram pouco efeito sobre o pH. A aplicação do herbicida não afetou o $\mathrm{pH}$ do solo.

Os resíduos de ambas as espécies diminuíram os teores de alumínio do solo, sendo que a ervilhaca foi mais eficiente. O principal mecanismo da redução do alumínio foi sua complexação por compostos orgânicos para a gramínea, e a complexação e a elevação do pH para a leguminosa.

Os resíduos vegetais aplicados sobre a superfície do solo aumentaram a eficiência do herbicida flumetsulam, especialmente quando aplicado sobre os resíduos da leguminosa, que teve maior efeito na elevação do $\mathrm{pH}$ do solo.
Os resultados obtidos neste trabalho sugerem que mesmo que a ação dos resíduos ocorra predominantemente na camada superficial e que seja de pouca duração, pode ser muito importante para o estabelecimento das culturas, pela redução do efeito tóxico do alumínio na fase inicial de crescimento das plantas, em que as raízes são mais sensíveis e, pelo aumento da atividade de herbicidas aplicados em pré-plantio.

\section{AGRADECIMENTOS}

Os autores agradecem ao pesquisador Eliséo Soprano da Estação Experimental da Empresa de Pesquisa Agropecuária e de Extensão Rural de Santa Catarina (EPAGRI)-Itajaí-SC pelo fornecimento do solo e suas respectivas análises de caracterização química e física.

\section{REFERÊNCIAS BILIOGRÁFICAS}

AMARAL, A.S. Reaplicação de calcário no sistema plantio direto consolidado. Porto Alegre, 1998. 102p. Dissertação (Mestrado em Ciência do Solo) - Programa de Pós-graduação em Agronomia, Universidade Federal do Rio Grande do Sul, Porto Alegre, 1998.

BAYLEY, G.W., WHITE, J.L. Factors influencing the adsorption, desorption and movement of peticides in soil. Residue Reviews, New York, v.32, n.1, p.29-92, 1970.

COMISSÃO DE FERTILIDADE DO SOLO - RS/SC. Recomendações de adubação e de calagem para os estados do Rio Grande do Sul e Santa Catarina. 3 ed. Passo Fundo: SBCS-Núcleo Regional Sul/EMBRAPA/CNPT, 1995. 224p.

ERNANI, P.R., GIANELLO C. Diminuição do alumínio trocável do solo pela incorporação de esterco de bovinos e camas de aviário. Rev Bras Ci Solo, Campinas, v.7, p.161-165, 1983.

FONTAINE, D.D., LECHMANN, R.G., MILLER, J.R. Soil adsorption of neutral and anionic forms of a sulfonamide herbicide, flumetsulam. Journal Environment Quality, Madison, v.20, n.4, p.759-762, 1991.

HARGROVE, W.L., THOMAS, G.W. Extraction of aluminum from aluminum-organic matter complexes. Soil Science Society American Journal, Madison, v.45, p.151-153, 1981.

HELYAR, K.R. The management of acid soils. In: WRIGHT, R.J., BALIGAR, V.C., MURRNÃN, R.P. Plant-soil interactions at low pH. Dordrecht : Kluver Academic, 1991. p.365-382.

HOYT, P.B., TURNER, R.C. Effect of organic materials added to very acid soils on $\mathrm{pH}$, aluminum, exchangeable $\mathrm{NH}_{4}$, and crop yields. Soil Science, Baltimore, v.119, p.227-237, 1975.

HUE, N.V., AMIEN, I. Aluminum detoxification with green manures. Comm Soil Sci PI Anal, New York, v.20, p.1499$1511,1989$.

HUE, N.V., CRADDOCK, G.R., ADAMS, F. Effect of organic acids on aluminum toxicity in sub-soils. Soil Science Society American Journal Soil, Madison, v.50, p.28-34, 1986. 
JUO, A.S.R, KAMPRATH, J. Copper chloride as an extractan for estimating the potentially reactive aluminum pool in acid soil. Soil Science Society American Journal, Madison, v.43, p.35-38, 1979.

KHAN, S.U. Soil organic matter. New York : Elsevier Science, 1978. The interaction of organic matter with pesticide. $319 \mathrm{p}$

KLESCHICK, W.A., GERWICK, B.C., CARSON, C.N. et al. DE-498, a new acetolactate syntase inhibiting herbicide with multicrops selectivity. Journal of Agricultural and Food Chemistry, Oxford, v.40, n.6, p.1083-1085, 1992.

LOCKE, M.A., BRYSON, C.T. Herbicide-soil interactions in reduced tillage and plant residue management systems. Weed Science, Champaign, v.45, n.2, p.307-320, 1997.
MIYAZAWA, M., PAVAN, M.A., CALEGARI, A. Efeito de material vegetal na acidez do solo. Rev Bras Cien Solo, Campinas, v.17, p.411-416, 1993.

MURPHY, G.P., SHAW, D.R. Field mobility of flumetsulam in three Mississipe soils. Weed Science, Champaign, v.45, n.4, p.564-567, 1997.

SALET, R.L. Toxidez de alumínio no sistema plantio direto. Porto Alegre, 1998. 109p. Tese (Doutorado em Ciência do Solo) - Programa de Pós-graduação em Agronomia, Universidade Federal do Rio Grande do Sul, Porto Alegre, 1998.

TEDESCO, M.J., GIANELlO, C., BISSANI, C., et al. Análise de solo, plantas e outros materiais. 2 ed. Porto Alegre: Depto de Solos da UFRGS, 1995. 174p. (Boletim Técnico, 5).

Ciência Rural, v. 30, n. 5, 2000. 